\title{
Comparación del aprendizaje, la actitud y el razonamiento científico en la enseñanza de la química a distancia y en la enseñanza tradicional
}

Alba Janeth Pinzón Rosas ${ }^{1}$

\section{Resumfen}

La presente investigación trata la comparación entre el aprendizaje, la actitud y el razonamiento cientifico de los estudiantes del curso de Química de dos universidades ubicadas en Bogotá, Colombia, una de ellas tiene el sistema de educación a distancia y en la otra la educación es tradicional, es decir, presencial. Las dos universidades tienen diferentes metodologías de enseñanza y los estudiantes de las dos son de estratos diferentes. La universidad a distancia es pública por lo que sus estudiantes son de estratos bajo y medio y la universidad presencial es privada y los estudiantes de allí pertenecen a estratos medio alto y alto. En la universidad a distancia hay cerca de 8 mil estudiantes y en la privada se cuenta con 2000 estudiantes.

El tipo de investigación elegido fue cuantitativo, bajo el cual sedesarrolló un diseño no experimental transeccional correlacional.

Los instrumentos se calificaron de acuerdo con las respuestas dadas por los estudiantes y su confrontación con las respuestas reales. LoS resultados se tabularon, utilizando intervalos de frecuencia para evitar colocar todos los datos obtenidos.

De acuerdo con los resultados se encontró que no hay diferencias significativas en ninguna de las variables utilizadas dependiendo de la modalidad de estudio. 


\title{
AlbajanthPinánPosess
}

Comparación del aprendizaje, la actitud y el razonamiento científico en la enseñanza de la química a distancia y en la

enseñanza tradicional, artículo producto de la Investigación

Batabras elave: Actitud, razonamiento científico, aprendizaje, educación a distancia, educación presencial

\section{A comparison of learning, attitude and scientific reasoning when teaching chemistry in distance and traditional education}

\begin{abstract}
This research compares students' learning, attitude and scientific reasoning in the chemistry course at two universities from Bogotá, Colombia. One of them has a distance education system and the other one follows a traditional education method. Both universities have different teaching methods and their students are different too.

The distance university is public and its students come from lower and middle strata. The other one is a private university, whose students come from upper middle and high strata. At the distance University there are about 8,000 students while the private one has 2,000 students. A ccording to the results, no significant differences werefound in any of the variables used depending on the mode of study.
\end{abstract}

Keywords: Attitude, scientific reasoning, learning, distance learning, classroom education.

Recibido: 11 Febrero de 2011

Aceptado: 18 Mayo de 2011

\section{lntroducción}

Con el proyecto desarrollado se pretende identificar si hay diferencias entre la actitud, el razonamiento científico y los conocimientos de los estudiantes dependiendo de la modali idad de estudio. Se realizo el estudio de estas tres caracterisțticas en dos, universidades con modalidades de estudio diferentes, a distancia y educacion presencial o tradicional. 
Revista de

investigaciones UNAD

Volumen 10. Número 1. Junio 2011

De acuerdo a la experiencia de los profesores de las dos universidades, se ha encontrado que en la enseñanza de la química se presentan inconvenientes en el aprendizaie y mucha apatia hacia el curso, los estudiantes de los programas que deben ver el curso aseguran que no les sirve para nada y su forma de razonar no es la esperada en este curso.

En estudios hechos en otras instituciones sobre el tema de la actitud se encontro que es negativa hacia los cursos de ciencia en particular con el curso de quimica. El razonamiento cientifico es muy pobre debido a que los alumnos no encuentran una relacion directa entre lo que estudian y el mundo que los rodea, por lo cual se presenta un problema y es que los estudiantes no sienten atracción hacia los cursos de ciencias natural es y creen que no son importantes dentro del programa academico que estudian.

Se respondió a la pregunta ¿Cómo cambia el aprendizaje, la actitud hacia la quimica y el razonamiento científico en los alumnos de la universidad a distancia en comparación con los que reciben educacion presencial?

En el marco teórico se incluye la descripción de las principales teorías en que se apoya la investigacion. Se presentan las mas relevantes para el tema de investigación. Se muestran las modalidades de educacion en las cuales se hara el estudio con sus principal es caracteristicas y las estrategias utilizadas en cada una de ellas, se mencionan las teorias utili izadas en la enseñanza de las ciencias, A continuación se habla del aprendizaje de la Química, del razonamiento cientifico y de la actitud.

El método describe el tipo de investigación elegido que es cuantitativo, bajo el cual se desarrolla un diseno no experimental transeccional correlacional, se explica el procedimiento para seleccionar la muestra y los criterios que se tomaron en cuenta para ello. Se presenta la técnica de recoleccion de datos junto con los instrumentos empleados para ello. Se describe el proceso de captura de los datos y como se analiza los datos obtenidos al aplicar los instrumentos que miden el aprendizaje, la actitud y el razonamiento científico.

Los resultados obtenidos al aplicar los instrumentos se presentan en tablas para cada test y para cada universidad. Estos resul tados se anal izan util izando los metodos estadísticos apropiados.

\section{Metodología}

Se hizo un estudio estadístico no experimental cuantitativo porque no se manipuló ninguna de las variables sino que se revisaron las situaciones ya 


\section{AlbaljanthPinónPosess}

Comparación del aprendizaje, la actitud y el razonamiento científico en la enseñanza de la química a distancia y en la

enseñanza tradicional, artículo producto de la Investigación

existentes en las dos universidades donde se realiza el estudio. Se toma un diseño transeccional correlacional.

Lapoblación con la que sellevó a cabo la investigación son los estudiantes del curso de Quimica general de primer semestre de 2009, de las universidades a distancia y de enseñanza tradicional donde labora la autora. El total de estudiantes es de 200.

Seestudiaronespecíficamentedos metodologías bajo las cuales sepuededesarrollar el aprendizaje como son la educacion a distancia y la educacion tradicional por lo tanto se tuvieron en cuenta los procesos y estrategias que son propias del desarrollo de cada una de las modalidades y especificamente que son empleadas estas universidades. Tambien se hicieron estudios del razonamiento cientifico de los estudiantes de las dos universidades y de su actitud hacia la química. Para evaluar los conceptos se util lizo el JCE Chemical Conceptual Questions. Permite saber la comprensión de conceptos vistos durante el semestre La prueba de conocimientos utili izada fue elaborada por la division de Educación Química de la Sociedad Quimica A mericana. Es una fuente de preguntas y problemas que se pueden ytil lizar en la enseñanza y la evaluacion de la comprension conceptual y la resolucion de problemas de quimica.

Los niveles de desempeño cognitivo se dividen, de acuerdo a la prueba de conceptos aplicada en: Interpretación de conceptos - Relacionar conceptos e imagenes - Busqueda de asociaciones entre preguntas y respuestas.

El test consta de 22 preguntas, y se califica de 1 a 100 siendo la nota entre 1 y 30 deficiente, entre 20 y 59 insuficiente, entre 60 y 75 , aceptable, entre 76 y 90 sobresaliente y mayor a 90 excelente, de acuerdo al metodo de calificación empleado en las dos universidades.

Para medir el razonamiento científico se utilizó el test de Lawson que ha sido diseñada para evaluar la capacidad de razonamiento científico de acuerdo a las, propuestas de Piaget. El test consta de 24 preguntas de respuestas de opcion multiple que miden seis aspectos del razonamiento: Conservacion de magnitudes física - Pensamiento de proporcionalidad - I dentificación y control de variables - Pensamiento probabilistico - Pensamiento combinatorio - Pensamiento correlacional.

Para la calificación de esta prueba es necesario que los estudiantes seleccionen tanto la respuesta correcta y la razón correcta. De acuerdo a Lawson (2007), si uno o ambos de estos son incorrectos, no se concede ninguna puntuacion, Hay veinticuatro preguntas en la prueba de Lawson, por lo que la puntuacion maxima que se puede ganar es de doce puntos. Para cuantificar la actitud se utilizo un test basado en el ASCI, (A tutitude-to-Subject-of-Chemistry Inventory), el test de Actitudes acerca de Ciencias, The Colorado Learning A ttitudes about Science Survey: Modification and Validation for U se in Chemistry, (Class Chem. 2009), 
Revista de

investigaciones UNAD

Volumen 10. Número 1. Junio 2011

modificado y validado para el uso en Química a los grupos de estudiantes. El test consta de 50 preguntas y tres opciones de respuesta por cada una de ell las, D: desacuerdo, A: de acuerdo, NS: no me interesa saberlo. En este caso se califico de 1 a 100 de forma que los puntajes obtenidos con notas entre 0 y 30 actitud negativa, entre 31 y 60 son indiferentes, entre 61 y 100 actitud positiva (Espinosa 1991).

Las fuentes que fueron consultadas para llevar a cabo la investigación son los estudiantes del curso de Química de la universidad a distancia y de la universidad de sistema tradicional para el primer semestre de 2009.

Los instrumentos se aplicaron al grupo de los estudiantes de cada una de las universidades en la primera semana de mayo, al final, del periodo academico correspondienteal primer semestreacademico. Seescogió un grupo al azar y previa lectura de las instrucciones se entrego a cada uno de ellos los test correspondientes

\section{Resultados}

Cada uno de los test se calificó numéricamente para poder realizar la estadística correspondiente.

A los datos obtenidos se halló la media y la varianza. Con la prueba de tde Student se calculo la diferencia de las medias de los resultados obtenidos en el test de razonamiento. El valor de thallado con la ecuacion es de 2. Tomando 100 grados de libertad y el valor hallado de t, de la tabla se obtiene una probabilidad de 0,025 . (2/2), Io que significa que no hay diferencia significativa entre las medias obtenidas para el test de razonamiento.

Con los resultados se comprueba la percepción de los profesores de las dos universidades respecto a la forma de razonar de los estudiantes. Solo, al gunos lo hacen de forma correcta porque no pueden ver de forma clara los fenómenos que ocurren a su al rededor y no los pueden relacionar con la quimica.

El valor de thallado con la ecuación es de 5. Tomando 100 grados de libertad y este valor de thallado se encuentra en la tabla de la distribucion t de Student que a/2 es de: 0.0010, lo que indica que la diferencia entre las medias de los resultados del test de actitud en las dos universidades no es significativa.

Las notas obtenidas son altas y de acuerdo a las preguntas realizadas, se puede inferir que los estudiantes tienen deseos de aprender quimica, el deseo de conocer y comprender, indagar en todas las cosas, la busqueda de datos y de su significado, veriticar las evidencias mediante experimentacion, hecho que contradice al gunos estudios realizados en los cuales la actitud hacia la química es negativa. 


\section{AlbaljanthPinónPosess}

Comparación del aprendizaje, la actitud y el razonamiento científico en la enseñanza de la química a distancia y en la

enseñanza tradicional, artículo producto de la Investigación

El valor de thallado con la ecuación es: 0,2. Para este valor y con 100 grados de libertad se observa que $\partial / 2$ es igual a 0,04, que indica que la diferencia de medias no es significativa. Los resultados evidencia la fal ta de claridad en los conceptos que tienen los estudiantes.

\section{Discusión de resultados}

Con la pregunta de investigación se quería saber si la metodología de estudio af ectaba de al guna manera estos tres aspectos Después de realizados los test de razonamiento, actitud y conceptos, de tabular, graficar los resultados y hacer el respectivo analisis se puede concluir, que la metodologia no influye en los resultados, los cuales son muy similares para las dos universidades y no hay diferencia significativa en las medias halladas utilizando la distribucion t de student.

La hipótesis inicial dada en cuanto a que el razonamiento de los estudiantes en la universidad de sistema tradicional era mejor al de los estudiantes de la universidad a distancia, se rechazo, al igual que la hipótesis inicial en cuanto a la actitud, es decir, no es cjerto que la metodología de estudio influya en la actitud de los estudiantes hacia de curso de quimica. . En cuanto a los conceptos tampoco se pudo comprobar que existan diferencias significativas de acuerdo a la modalidad de estudio.

De acuerdo a los objetivos mencionados, se puede decir que se al canzó a cumplir con ellos, el impacto de la educacion y las diferencias académicas se midieron con el test de conceptos, en el cual los puntajes obtenidos por estudiantes de las dos universidades fue muy similar. Es de notar que este promedio es muy bajo lo cual es preocupante.

En cuanto al análisis de la actitud de los estudiantes, se observó que los resultados contradicen al gunos estudios acerca del tema. El promedio en esta prueba es alto para las dos universidades. Se eval uo el razonamiento, que es uno de los objetivos especificos y se encontro que al igual que en el test de conceptos los resultados promedio son muy bajos.

\section{Conclusiones}

Las implicaciones de los resultados de esta investigación son las siguientes:

Para lograr que los estudiantes al cancen una formación científica es necesario despertar su interes y profundizar en los conceptos químicos y su aplicacion 


\section{Revista de \\ investigaciones UNAD \\ Volumen 10. Número 1. Junio 2011}

a situaciones concretas, relacionándolos con la natural eza, con el fin de que comprendan la utilidad de los conocimientos conceptuales y se llegue a un aprendizaje significativo.

Si se desarrollan estrategias innovadoras de enseñanza-aprendizaje de las ciencias en las aulas tanto del sistema tradicional como del sistema a distancia se puede conseguir que los estudiantes tengan un mejor desempeno academico y sus resul tados en las evaluaciones sean mejores.

Es necesario mantener a los estudiantes motivados para que de esta manera logren una mejor aprehension de los temas del curso.

\section{Referencias bibliográficas}

Class Chem.(2009). The Colorado Learning Attitudes about Science Survey: Modification and Validation for Use in Chemistry. Journal of Chemical Education, 85, (10).

Cigic, L. (2006). How to Motivate Students to Study before They Enter the Lab Pogacnik, Journal of Chemical Education; 83(7); p. 1094. (ERIC Document Reproduction Service No.EJ 820295).

Coletta, V. P.; Phillips, J. A. y Steinert, J. J. (2007). Why you should measure your students' reasoning ability. The Physics Teacher, 45(4), 235-238.

De J ong, O. (1996). La investigación activa como herramienta para mejorar la enseñanza de la Química. Revista enseñanza de las ciencias.14(3),pp. 279-288. Universidad de Utrech. Distrito Escolar Unificado De Riverside Departamento de Educación.

Lawson, A. (2007). The development and validation of a classroom test of formal reasoning. J ournal of Research in Science Teaching, 15 (1)

Pogacnik, Journal of Chemical Education; 83(7); p. 1094. (ERICDocument Reproduction Service No.EJ 820295).

Vargas, M. ( 2005) Educación y formación del pensamiento científico Journal of Science Education. 6,(1), p.51Recuperado el 13 de febrero de 2009 desde http://www . bookrags.com/highbeam/educacin-y-formacin-del-pensamiento-hb/ 